\title{
Erratum to: Homogenization method for one-dimensional photonic crystals with magnetic and chiral inclusions ${ }^{\star}$
}

Eur. Phys. J. B (2020) 93: 124, https://doi.org/10.1140/epjb/e2020-10095-4

Javier Flores Méndez ${ }^{1,2}$, Benito Zenteno Mateo ${ }^{1}$, Mario Moreno Moreno ${ }^{3}$, Alfredo Morales-Sánchez ${ }^{3}$, Gustavo M. Minquiz ${ }^{1,2}$, Hector Vázquez Leal ${ }^{4,5}$, Israel Vivaldo-De la Cruz ${ }^{1}$, Silvia Cortés-López ${ }^{1}$, Ana Cecilia Piñón Reyes ${ }^{1}$, and Roberto Ambrosio ${ }^{1, a}$

${ }^{1}$ Benemérita Universidad Autónoma de Puebla-Ciudad Universitaria, Blvd. Valsequillo y Esquina, Av. San Claudio s/n, Col. San Manuel, C.P. 72570, Puebla, Pue, Mexico

2 Tecnológico Nacional de Mexico/I.T. Puebla-División de Estudios de Posgrado e Investigación, Av. Tecnológico No. 420, Maravillas, C.P. 72220, Puebla, Pue, Mexico

${ }^{3}$ Instituto Nacional de Astrofísica, óptica y Electrónica, Luis Enrique Erro No. 1, C.P.72840, Sta. Ma. Tonantzintla, Puebla, Pue, Mexico

${ }^{4}$ Facultad de Instrumentación Electrónica, Universidad Veracruzana, Cto. Gonzalo Aguirre Beltrán s/n, 91000 Xalapa, Veracruz, Mexico

${ }^{5}$ Consejo Veracruzano de Investigación Científica y Desarrollo Tecnológico (COVEICYDET), Av. Rafael Murillo Vidal No. 1735, Cuauhtémoc, 91069 Xalapa, Veracruz, Mexico

Received 3 July 2020

Published online 3 August 2020

(C) EDP Sciences / Società Italiana di Fisica / Springer-Verlag GmbH Germany, part of Springer Nature, 2020

Reference 42 is corrected to:

42. T.G. Mackay, A. Lakhtakia, SPIE Rev. 1, 018003 (2010)

\footnotetext{
${ }^{\star}$ The online version of the original paper can be found at https://doi.org/10.1140/epjb/e2020-10095-4

${ }^{a}$ e-mail: roberto. ambrosio@correo.buap.mx
} 